\title{
DESIGNING THE MINIMUM DATA SET OF PSYCHIATRIC EMERGENCY RECORD
}

\author{
Type of article: conference abstract
}

\author{
Zahra Ebnehoseini1, Marziyhe Meraji2*, Farzad Akbarzadeh3 and Malihe Irajzade4 \\ 1: Ph.D. Student Medical Informatics, Department of Medical Informatics, School of \\ Medical, University of Medical Sciences, Mashhad, Iran. \\ 2: Ph.D. in Health Information Management, Assistant Professor, Department of Medical \\ Records and Health Information Technology, School of Paramedical Sciences, Mashhad \\ University of Medical Sciences, Mashhad, Iran. \\ 3: Psychiatrist, Assistant Professor of Psychiatry, Psychiatric and Behavioral Sciences \\ Research Center, Mashhad University of Medical Sciences, Mashhad, Iran. \\ 4: Masters in Clinical Psychology, Psychiatric and Behavioral Sciences Research Center, \\ Mashhad University of Medical Sciences, Mashhad, Iran. \\ * Tel: +98.09370802011, E-mail: MerajiM1@mums.ac.ir
}

\begin{abstract}
Introduction: Psychiatric emergencies are acute mental health disturbances, behavior and social relationship that require immediate intervention. The major role of psychiatric emergency services is to provide mental health care services for patients with acute mental health problems. Design emergency psychiatry core dataset has improved the coordination and integration of services and improved the outcomes for the patient with severe and persistent mental illness with complex needs. So the aim of this study was to design data elements (DEs) in emergency psychiatry for Iran.

Methods: This is an applied study. Emergency psychiatry (DEs) collected via literature review and then psychologist and psychiatrist (16 experts) assign the score from 0 to 5 to them according to the value of each data element. (DEs) selected as core Emergency psychiatry (DEs) that were achieved 4 or 5 scores from $75 \%$ specialist.

Results:According to the literature review, 110 (DEs) included studying. 13 experts (8 psychologists, 8 Clinical Psychologist) evaluated psychiatric emergency (DEs) set. The average work experience of psychiatrists and psychologists was 16 years and their work experience ranged from 2 to 25 years (table 1). according to the experts opinion, 54 (DEs) with at least $75 \%$ of the agreement were identified as the psychiatric emergency (DEs). Emergency psychiatric (DEs) and average agreement of each of them were: demographic characteristics (6 DEs with an agreement average of $82.5 \%$ ), history of mental illness (9 DEs with an agreement average of $79 \%$ ), family history of psychology (3 DEs with an average agreement of 77.08\%), medical history (1 DEs with an average agreement of $81.25 \%$ ) Assessment of mental status ( 20 DEs with an average agreement of $82 \%$ ), assessment of the self harm risk or harm risk for others ( 13 DEs with an average agreement of 93.6\%) and diagnosis and treatment (3 DEs with an average agreement of $81.25 \%)$.

Conclusion:Given the importance of psychiatric disorder and lack of the national system for gathering psychiatric information, perform the same study abut psychiatric data element is very important. The results of this study can be used for design psychiatric emergency forms and gather accurate and complete patient information.

KEYWORDS: Minimum Data Set, Psychiatric Emergency, Mental Health
\end{abstract}


Medical Technologies Journal, Volume: 1, Issue: 4, October-December 2017, Pages:76-136. Doi : https://doi.org/10.26415/2572-004X-vol1iss4

\section{Declaration of conflicts}

This abstract is selected from the First International Congress of Diseases and Health Outcomes Registry and First National Congress of Medical Informatics, 14-17 February 2017, Mashhad, Iran

\section{Authors' biography}

No biography.

\section{References}

No references. 